\title{
Severe Low Back Pain as The Presenting Feature of Alkaptonuria in A Young Female
}

\author{
Archana Shetty ${ }^{1 *}$, Padma Priya Kasukurti ${ }^{1}$, Hariprakash², and Vijaya $\mathbf{C}^{1}$ \\ ${ }^{1}$ Dept of Pathology, Sapthagiri Institute Of Medical Sciences \& Research Center, Bangalore, India \\ ${ }^{2}$ Dept. of Neurosurgery, Sapthagiri Institute Of Medical Sciences \& Research Center, Bangalore, India
}

\begin{abstract}
Alkaptonuria is a rare inborn error of metabolism, caused due to the deficiency of the enzyme homogentisic acid oxidase. The manifestations of this disease are initially non specific, hence the condition is often under diagnosed as the patients present at later stages with associated complications. Although the treatment of alkaptonuria is mainly supportive, the diagnosis and reporting of this rare condition is necessary as it avoids subjecting the patients to unnecessary investigations and interventions.

We report a case of a thirty five year old lady, who presented with severe low back pain. X ray showed features of disc prolapse. On table intraoperative findings showed blackish discolored disc material during discectomy. After doing simple ancillary tests, and correlating with other clinical features, the lady was diagnosed as a case of Alkaptonuria.

We report this case as not only is the metabolic condition rare, but also to highlight the importance of simple urinary tests which are of great help in diagnosing such conditions, even in this era of automation considering the cost effectiveness in rural population like ours.
\end{abstract}

Keywords: Low Back Pain, Urine Examination, Homogentisic Acid, Oochronosis, Alkaptonuria

\section{Introduction}

Alkaptonuria is a rare, inborn error of metabolism. It is an autosomal recessive condition caused due to the deficiency of the enzyme homogentisic acid oxidase. It was also one of the four inborn errors of metabolism described by Garrod in his croonian lectures of 1908. [1] The common clinical manifestations of alkaptonuria are (i) homogentisic aciduria, (ii) ochronosis (deposition of bluish-black pigment in all connective tissues), and (iii) arthritis.

[2]. Though no specific treatment is available till date, there is need to diagnose and report this condition as patients' survival can be improved by dietary modifications and other ancillary treatments.

\section{Case Report}

A thirty five year old female was admitted with history of severe low back pain and stiffness since the past two months, which aggravated since a week. The backache was of gradual onset initially, reaching to a severity that she was not able to get up from the sitting position without support at the time of presentation. The patient had no other significant complaints. She also gave history of being operated in ankle at the region of the Achilles tendon two years back, but no documents of the same were available. The patient had no significant family history.

General examination showed brown to black pigmentation of the ear cartilage and the anti helix.
[FIG 1]The interpalpebral fissures of both eyes showed light brownish pigmentation.

$\mathrm{X}$ ray of the spine showed extensive calcifications of the intervertebral disc spaces and osteophytosis. Disc prolapse was noted at the level of L3 - L4 spine. Also noted was

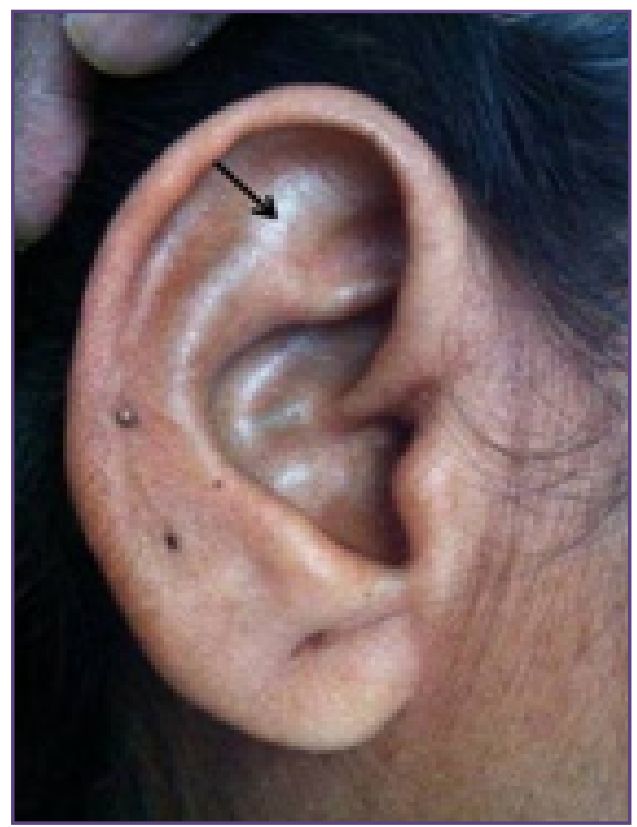

Fig. 1: Ear showing light brownish pigmentation in the anti helix region (Arrow). 
mild osteoarthritis of the knee and hip joints on $\mathrm{X}$ ray of these joints. In view of disc prolapsed a discectomy was planned. Intra operative findings showed brown to blackish pigmentation of the disc material.The scooped disc material was sent for histopathological examination.[FIG2A]. Histopathological examination showed fragments of mature cartilage showing mild degenerative changes and brownish pigmentation at places.[FIG 2B]

Laboratory investigations:Simultaneously, other laboratory investigations were done. Routine biochemical parameters including the ECG were normal. However taking into consideration the clinical findings and the disc material discoloration, Alkaptonuria was suspected. The patient's urine sample on standing for twenty four hours turned black in color [FIG3A]. Benedict's test done on the urine sample showed a brick red precipitate. A black precipitate was observed on doing ammoniacal silver nitrate test. Ferric chloride test done on urine showed a transient dark green colour. [FIG3B]

The patient was thus diagnosed as a case of Alkaptunuria based on the clinical and other investigation findings. She was given required doses of vitamin $C$, and advised dietary modification to avoid phenylalanine and tyrosine rich foods. Symptomatic treatment for joint pains was given with analgesics, and sessions of physiotherapy was advised. The patient, being from a rural background, was eventually lost to follow up.

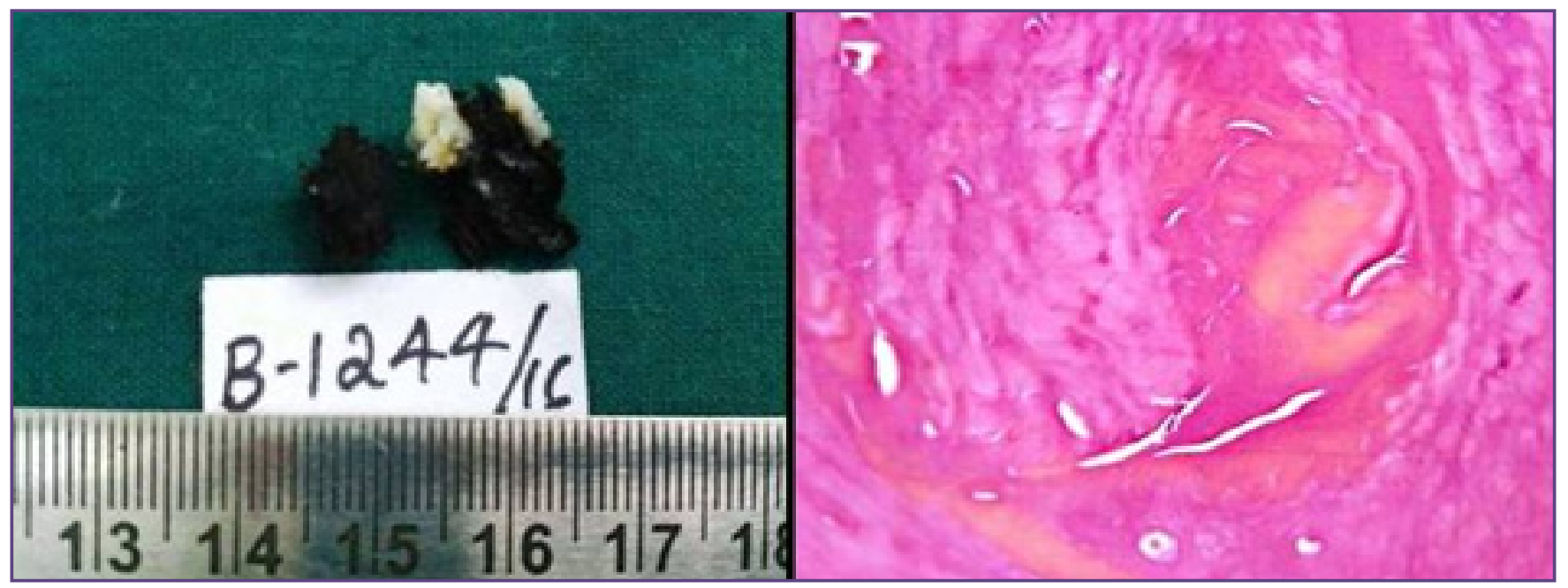

Fig. 2 A: Scooped out disc material sent for histopathological examination .Fig 2B: Photomicrograph showing cartilaginous bits with foci of brownish pigmentation, H\&E X100.

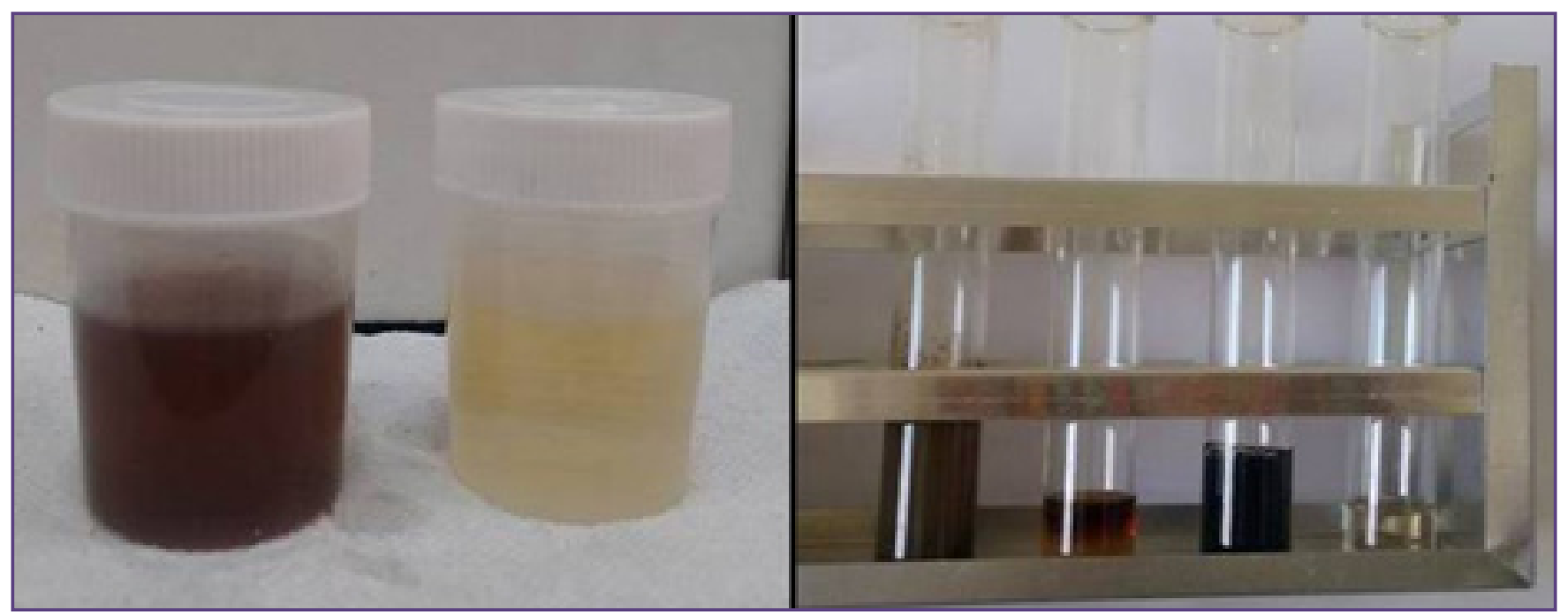

Fig. 3A: Urine sample after standing for 24 hours (right) as compared to fresh sample(left).FIG 3B: Urine sample showing control sample, Ferric chloride test , Benedicts test and silver nitrate test (left to right). 


\section{Discussion}

Alkaptonuria [AKU] is a rare autosomal recessive metabolic disorder, characterized by the deficiency of homogentisic acid oxidase, an enzyme in the metabolic pathway of phenylalanine and tyrosine. It also is credited with being one of the first diseases for which the laws of Mendelian inheritance was proposed..$^{[1]}$ The deficiency of homogentisic acid oxidase leads to the accumulation of a melanin like granular pigment, which has affinity for cartilage, and resembles ochre (yellow), hence the name oochronosis. ${ }^{[3]}$

Although one of the earliest manifestations of this disease is the darkening of urine, this is seldom the presenting complaint as patients do not observe the same. Our patient also did not notice any change in the colour of urine. However, her urine sample on standing turned dark after twenty four hours during urine examination. Benedicts test, a non specific test for reducing substances is positive for homogentisic acid. Sachadevet $\mathrm{al}^{\left[{ }^{[4]}\right.}$ have reported a case of alkaptonuria, misdiagnosed initially as diabetes mellitus on basis of Benedicts test alone. The patient was given hypoglycaemic drugs, after which he went into hypoglycaemic coma. This situation is however uncommon in current practice as dipsticks, which specifically test glucose are used in the clinical laboratories. We would also want to highlight the importance of simple, inexpensive but valuable tests like ferric chloride and the silver nitrate test, which can detect the homogentisic acid in urine quantitatively and are highly cost effective, considering the rural population of our country. ${ }^{[5]}$

The major weight bearing joints of the body get affected after the third decade of life in Alkaptonuria , most common being the intervertebral discs of the lumbar spine, hip and the knee. ${ }^{[6]}$ Low back pain is one of the commonest presenting symptoms in young patients. The deposition of pigment happens in these joint $\mathrm{s}$ as the ochronotic pigment has a very high affinity for collagen fibers of the joints. ${ }^{[7]} \mathrm{As}$ most of the patients present with non specific symptoms of low back pain and stiffness of joints, the metabolic defect is often masked, as in our case. ${ }^{[8]}$ Degenerative arthritis and ankylosing sponylitiis [AS] are the close differentials to be considered. Annular sclerosis is a feature of AS, unlike AKU. The sacro - iliac joints in AKU show narrowing and marked sclerosis, which is not seen in AS. ${ }^{[9]}$ Intra operative findings of blackish discolored disc material as seen in our case, provided us some clue to work up for alkaptonuria. On histopathological examination the ochronotic pigment needs to be differentiated from melanin. Ochronotic pigment does not stain with silver nitrate, and blackening on methylene blue or cresyl violet staining is a useful method to highlight it. ${ }^{[10]}$
Spontaneous rupture of the Achilles tendon is also an important clinical manifestation of Alkaptonuria. Deposition of the pigmented polymers weakens the structural integrity of the tendons causing their rupture. Most cases of Achilles tendon rupture till date have been reported from India. ${ }^{[9,11]}$ Interestingly even our patient gave a similar history of having some surgical correction in the region of achilles tendon, but no documents or discharge summary of the same was available for confirmation.

Renal, prostatic, salivary stone formation, cardiovascular manifestations ( valvular calcification, coronary artery calcification, aortic stenosis), rupture of ligaments are some of the documented complications of Alkaptonuria. $[2,8,12]$. There is no definitive treatment for this condition till date. The management strategies are directed towards early diagnosis of this condition and timely intervention to delay the complications. Ascorbic acid is shown to inhibit the binding of homogentisic acid to the chondrocytes, hence the ideology in giving patients supplementation of the same in doses of $1 \mathrm{~g}$ /day. Nitisinone an inhibitor of 4 - hydroxyphenylpyruvate, the enzyme that produces homogentisic acid has also been tried in these patients. A recent study by Millucci et al ${ }^{[13 \mid}$ provided experimental evidence that AKU osteoarticular tissue contains AAamyloid deposits . This opens new perspectives for AKU therapy. The study also showed that methotextrate was able to significantly prevent in vitro HGA-induced A-amyloid aggregates, thereby contributing new perspectives to the clinical picture of this disease.

\section{Conclusion}

Alkaptonuria is a rare metabolic disease, which is often underdiagnosed as patients often present at later stages with associated complications. Though no specific treatment has been discovered till date, the importance of recognizing this condition lies in helping the patients delay the onset or tide over the already manifested complications. Also, diagnosis of this metabolic disease with simple urine tests can prevent the patient from being subjected to unnecessary medications and interventions in this era of automation.

\section{References}

1. Pratibha K, Seenappa T, Ranganath K. Alkaptonuric ochronosis: Report of a case and brief review. Indian Journal of Clinical Biochemistry. 2007;22(2):158-161.

2. Wilke A, Steverding D. Ochronosis as an unusual cause of valvular defect: a case report. Journal of Medical Case Reports. 2009;3:9302.

3. Babanagare SV, Deshmukh SD, Khadilkar MS, Patil AA. Ochronosis: A report of three cases and review of the literature. Indian J Pathol Microbiol 2011;54:626-8. 
4. Sachdeva J R, Gupta M M, Jindal V K. Alkaptonuria (a case report). J Postgrad Med 1986;32:166-7,170A.

5. Singh O, Muthukrishna Pandian R, Sudhakar Kekre N Alkaptonuric Ochronosis.

6. Urology. 2017 Feb;100 :e3-e4

7. Gil JA, Wawrzynski J, Waryasz GR Orthopedic Manifestations of Ochronosis: Pathophysiology, Presentation, Diagnosis, and Management. Am J Med. 2016 May;129(5):536.e1-6

8. Craide FH, Fonseca JSBM, Mariano PC, Fernandez NM, Castro CGC, Mene YSL. Alkaptonuria - Case report. An Bras Dermatol. 2014;89(5):799-801

9. Li N, Tian W, Yuan Q, He D. Cervical Spondylotic Myelopathy due to the Ochronotic Arthropathy of the Cervical Spine. Journal of Korean Neurosurgical Society. 2016;59(1):65-68.
10. Rathore FA, Ayaz SB, Mansoor SN. Ochronotic Arthropathy: Two Case Reports from a Developing Country. Clinical Medicine Insights Arthritis and Musculoskeletal Disorders. 2016;9:15-20.

11. Vallonthaiel AG, Kakkar A, Singh MK1, Ramam M. Ochronosis with subtle histological findings Indian J Dermatol Venereol Leprol. 2015 Nov-Dec;81(6):623-4

12. Alajoulin OA, Alsbou MS, Ja'afreh SO, Kalbouneh HM. Spontaneous Achilles tendon rupture in alkaptonuria. Saudi Medical Journal. 2015;36(12):1486-1489.

13. Panjwani PK, Kini U, Choubey SK, Mohan A. Coexistence of Alkaptonuric Ochronosis and renal cell carcinoma: An unusual association and report of a case. Indian $\mathrm{J}$ Pathol Microbiol 2015;58:255-6

14. Millucci L, Spreafico A, Tinti L, et al. Alkaptonuria is a novel human secondary amyloidogenic disease. Biochimica et Biophysica Acta. 2012;1822(11):1682-1691.

*Corresponding author:

Dr. Archana Shetty, Associate Professor, Pathology, FF3, Mythili, Sai Durga Apartments, Padmavathi Meenakshi Kalyana Mantapa Road,

Rajarajeshwarinagar, Bangalore(India) 560098

Email: archanashetty2924@gmail.com

Date of Submission : 07.02.2017

Date of Acceptance : 27.06.2017

Financial or other Competing Interests: None.

Date of Publication : 19.10.2017 